\title{
Gamificación e Informática. Comparativa de una experiencia mixta en Grado y Máster.
}

\section{Juan Vicente Oltra Gutiérrez ${ }^{\mathrm{a}}$}

${ }^{a}$ Escuela Técnica Superior de Ingeniería Informática. Universitat Politècnica de València. jvoltra@omp.upv.es

\begin{abstract}
The same gamification experience is applied in two similar subjects in terms of their contents and both with a reduced number of students, where the fundamental difference between them lies in the characteristics of the student, because while one is developed in the Double Degree in Computer Science and Business Administration, the other one in Master.

Intuitively, better results were expected a priori with a student that is usually classified as "more mature". In this paper, we try to compare the results obtained .
\end{abstract}

Keywords: gamification, methodology, evaluation.

\begin{abstract}
Resumen
Se aplica la misma experiencia de gamificación en dos asignaturas de corte similar en lo que respecta a sus contenidos y ambas con un número de alumnos reducido, donde la diferencia fundamental entre las mismas radica en las características del alumno, pues mientras una se desarrolla en el Doble Grado de Informática y Administración de Empresas, la otra lo hace en Máster.

De forma intuitiva se esperaba a priori unos resultados mejores con un alumnado que suele ser catalogado como "más maduro" En el presente trabajo se trata de comparar los resultados obtenidos.
\end{abstract}

Palabras clave: gamificación, metodología, evaluación.

\section{Introducción}

La percepción de la madurez de los alumnos, de cómo éstos cambian su actitud ante los estudios conforme se van cargando de años y experiencia, ha sido estudiada desde múltiples prismas y en distintos escenarios (Boza, 2012); (Creed, 2012); (Donoso, 1998); (Kinash, 2017).

Aun sin necesidad de recurrir a análisis centrados en la percepción obtenida por los docentes, en cualquier conversación informal surge como idea el que los alumnos que cursan estudios de máster son más constantes y se preocupan más por su propio aprendizaje que sus compañeros de grado. A título personal esta idea preconcebida de diferencia de 
grados de maduración, sin poder constatar mediante herramientas científicas la misma, me ha servido para hacer diseños diferentes para asignaturas en distintos años. Con el presente trabajo se trata de cuantificar esa la constancia del alumnado, en dos asignaturas que de forma intencional han mantenido un cierto paralelismo (en la medida en que lo permite la diferencia entre las mismas), de forma en que se nos permita establecer comparaciones. Ambas asignaturas además tienen un perfil similar de contenidos (aunque una profundiza más que la otra en determinadas materias), transcurren en el mismo semestre del mismo curso, y sobre ambas se aplica una misma técnica específica, de forma que los resultados, presentando la lógica disparidad por sus diferentes orígenes, permitan elaborar una comparación del seguimiento que los alumnos han hecho de las mismas.

\section{Definición del escenario:}

Las asignaturas a comparar son:

- Marco Legal y Deontológico (Máster Universitario en Gestión de la Información), código 34015, en adelante MLD

- Deontología y Profesionalismo (Doble grado en Informática y Administración de Empresas), código 11550, en adelante DyP

Ambas comparten una serie de características comunes que las hacen idóneas para la comparación. Por una parte sus contenidos son de corte similar, aunque obviamente en niveles distintos de complejidad. Ambos temarios descansan en la deontología informática y en los aspectos legales básicos de los sistemas de información. También la extensión es similar, pues ambas tienen una carga teórica de tres créditos. Además, en ambos casos el número de alumnos matriculados es reducido. Al describir la experiencia delimitaremos el conjunto de semejanzas y disimilitudes de una forma más precisa.

Para poder establecer la comparación, usamos una técnica empleada desde hace varios cursos en ambas asignaturas, ya consolidada en procedimientos y de uso uniforme en ambos casos: usaremos la llamada "gamificación", descrita en versiones anteriores (Oltra 2016a); (Oltra, 2016b). De igual modo, de forma sucinta delimitaremos los cauces por los que transcurre.

\section{Objetivos}

Vamos a comparar una serie de resultados, algunos obtenidos de la observación directa, y otros de herramientas automatizadas, que nos van a permitir observar diferencias y analogías en el desarrollo de una actividad concreta, la "gamificación”.

Conviene fijar el punto de partida, definiendo que entendemos por "gamificación", un término que emplearemos con asiduidad y del que, por comodidad, a partir de este momento prescindiremos de las comillas.

El término gamificación (gamification) vino de la mano de Nick Pelling (Pelling, 2011)], un programador y desarrollador de videojuegos que trato de lograr que las interfaces no relacionadas con juegos se parecieran más a los juegos, para lograr unas transacciones divertidas y rápidas" conocidos mundialmente. El término aparece en 2002, pero comenzó a

(cc) EY-NC-ND 2018, UniversitatPolitècnica de València

Congreso IN-RED (2018) 
ganar vuelo cuando se popularizó por las empresas y eventos científicos, pudiendo definirse como "El uso de elementos de diseño de juegos en contextos no relacionados con juegos".(Deterding et al., 2011), o, (Werbach, 2014), "El proceso de hacer actividades más parecidas a juegos"

Siguiendo trabajos precedentes (Oltra 2016a y Fuchs, 2014), en este contexto empleamos la palabra y sus derivados por su extenso uso dentro de la comunidad docente, evitando otros con menos éxito en la misma, como ludificación o juguetización. Entenderemos como tal el uso de, elementos y dinámicas propias de los juegos en actividades no lúdicas con el fin fijado en el apoyo al aprendizaje, siendo un elemento a destacar en este tipo de actividades una serie de recompensas otorgadas una vez se alcanzan pequeños logros.

Cabe indicar desde este momento, para evitar la confusión con el aprendizaje basado en juegos (en inglés, game-based learning), fenómeno próximo en apariencia a este que nos ocupa de la gamificación, las diferencias principales con la experiencia que aquí se describe. El aprendizaje basado en juegos está clasificado como una rama de los juegos que se ocupa de asumir los objetivos de aprendizaje desde otros entornos. Y precisamente nosotros no cambiamos de entorno, puesto que lo único que adoptamos del mecanismo del juego es la competición, su dinámica. No creamos un juego propio ni tan siquiera adaptamso un juego pre-existente. No buscamos tan siquiera enseñarles a jugar. Se mantiene una serie de semejanzas, como sus metas principales centradas en la resolución de muchos de los problemas que se dan en el aula, tales como la falta de atención, desmotivación, etc., pero estas metas son suficientemente comunes con tantas estrategias alternativas como para desechar una equiparación por las mismas.

A pesar de que se emplea la gamificación desde hace cinco semestres, para esta ocasión se ha simplificado, en aras de facilitar la toma de datos. La descripción exhaustiva de la experiencia original puede verse en (Oltra, 2016b), pero para esta ocasión ha quedado reducida a tres vías:.

- Uso de Twitter. Desde la cuenta del profesor, se mantiene un contacto con los alumnos, voluntario por su parte. Entre los tuits emitidos se cuentan algunos acompañados de un hastag con las siglas de la asignatura (\#DyP; \#MLD) que contienen noticias o comentarios relativos a temas vistos o próximamente a ver en las sesiones de la asignatura. Los alumnos responden a los mismos y se "premia" el mejor comentario (texto aclaratorio, enlace o desmentido si la noticia resulta ser falsa).

- Uso de los foros de la asignatura. Usando las herramientas que nos ofrece la plataforma Poliformat de la Universitat Politécnica de Valencia, se generan debates online sobre casos vistos en la asignatura, con una duración de una semana. Las mejores participaciones son premiadas.

- Debates en aula. Algunas de las sesiones presenciales terminan con un debate de aula sobre un caso real que se analiza a la luz de los contenidos teóricos de la asignatura. Estos casos darán paso a tareas que deben realizar o test online que deben responder. Pero durante el desarrollo del debate estos se animan "gamificándolos" empleando a tal fin unos billetes falsos, como los que se 
emplean en juegos de mesa tales como el Monopoly, que, al final de la sesión, son recogidos para ser convertidos en calificaciones. Puede verse que es esta la única vía donde el alumno conoce inmediatamente su resultado de participar en la actividad.

Para animar la competición, se fija un tope, un máximo a alcanzar, y se premia a los primeros en llegar a él, tanto socialmente, reconociéndolo en clase, como con una mínima puntuación extra. Ese tope se ha fijado en un (1) punto.

Y una vez descrito el camino a seguir, nos queda describir a los participantes. Hay factores cualitativos y otros cuantitativos a tener en cuenta $\mathrm{y}$, aunque los primeros pequen de subjetividad no cabe orillarlos pues el hacerlo nos provocaría una visión parcial de la experiencia.

\subsection{Participantes:}

El perfil de los alumnos y sus variables que pueden ser cuantificados de forma numérica puede verse en la tabla 1 .

Hagamos notar una observación antes de ver la tabla: el número de participantes en MLD se cifra en 20, y no 21, que es el número de matrícula, pues el alumno que falta no solo no vino a una sesión de la asignatura, sino que era un completo desconocido para sus compañeros.

Para poder efectuar visualmente las comparaciones, al lado del número bruto de estudiantes indicado en cualquier característica, se indica el porcentaje que ese número resulta del total de alumnos en la asignatura. Esta representación se repite en las tablas siguientes.

Tabla 1. Características de los participantes.

\begin{tabular}{|c|c|c|c|c|}
\hline Asignatura / características de los alumnos & DyP & & MLD & \\
\hline Número de alumnos reales & 29 & & 20 & \\
\hline $\begin{array}{l}\text { Alumnos españoles o residentes permanentes en } \\
\text { España }\end{array}$ & 29 & $\begin{array}{l}100 \\
\%\end{array}$ & 10 & $50 \%$ \\
\hline $\begin{array}{l}\text { Alumnos de otras procedencias (Rusia, Colombia...) } \\
\text { que residen en el país para cursar sus estudios }\end{array}$ & 0 & $0 \%$ & 10 & $50 \%$ \\
\hline Alumnos que simultanean trabajo con estudios & 1 & $\begin{array}{l}3,5 \\
\%\end{array}$ & 6 & $30 \%$ \\
\hline Rango de edad (años) & $20-21$ & & $22-36$ & \\
\hline Alumnos con conocimientos previos de la materia & 0 & $0 \%$ & 5 & $25 \%$ \\
\hline $\begin{array}{l}\text { Alumnos emancipados (viven solos o con su familia, } \\
\text { asumiendo una parte significativa de los gastos de la } \\
\text { vivienda, sin financiación familiar) }\end{array}$ & 0 & $0 \%$ & 3 & $15 \%$ \\
\hline
\end{tabular}

Fuente: Elaboración propia 


\subsection{Asignaturas:}

En la tabla 2 vemos las diferencias más significativas entre las asignaturas. A continuación de la tabla revisamos esas diferencias para matizar en qué modo pueden afectar a la comparación.

Hagamos notar que en las 10 sesiones de MLD se incluyen tanto las sesiones de presentaciones de trabajos. Los trabajos, obligatorios en MLD, adquirían la característica de voluntarios en DyP, sirviendo para que aquellos alumnos en el límite de una calificación a otra superior, pudieran dar el salto. En este semestre se presentaron tan solo dos trabajos.

Tabla 2. Características de las asignaturas.

\begin{tabular}{|c|c|c|}
\hline Asignatura / características & DyP & MLD \\
\hline Créditos totales de teoría & 3 & 3 \\
\hline Créditos totales de práctica & 1,5 & 0 \\
\hline Inicio de clases & 5/sept & $10 /$ nov \\
\hline Fin de clases & 22/dic & 20/dic \\
\hline Duración de una clase de teoría (en horas) & 2 & 3 \\
\hline $\mathrm{N}^{0}$ de clases de teoría a la semana & 1 & 2 \\
\hline $\mathrm{N}^{0}$ de clases de teoría en el semestre & 13 & 10 \\
\hline $\mathbf{N}^{0}$ de tareas que se evalúan & 3 & 5 \\
\hline Peso de las tareas en la nota de la asignatura & $30 \%$ & $50 \%$ \\
\hline $\mathrm{N}^{0}$ de casos de aula con test que se evalúan & 5 & 2 \\
\hline Peso de los casos evaluados en la nota de la asignatura & $35 \%$ & $20 \%$ \\
\hline $\mathrm{N}^{0}$ de casos de aula totales que se realizan & 7 & 5 \\
\hline Se presenta trabajo de asignatura & Voluntario & SI \\
\hline Peso del trabajo en la nota de la asignatura & $0 \%$ & $30 \%$ \\
\hline $\mathrm{N}^{\circ}$ de trabajos de prácticas que se evalúan & 4 & 0 \\
\hline Peso de los trabajos de prácticas en la nota de la asignatura & $35 \%$ & $0 \%$ \\
\hline Gamificación. Foros abiertos para debate & 7 & 2 \\
\hline Gamificación. Foros empleados realmente para debate & 1 & 2 \\
\hline Gamificación. Semanas de actividad en Twitter & 10 & 5 \\
\hline Gamificación. Debates de aula & 7 & 5 \\
\hline
\end{tabular}


Vemos que no se trata de dos desarrollos idénticos, pero observemos cuales son las diferencias para tratar de señalar aquellos puntos que puedan distorsionar la comparación.

- Tamaño en créditos de la asignatura: observamos que en la asignatura de grado existe una parte práctica de la que carece la asignatura de Máster. Dado que las actividades de gamificación se practican exclusivamente en la parte teórica, este elemento no nos afecta.

- Sesiones de teoría: la diferencia es evidente, tanto en número y tamaño (menos sesiones y de más duración para el máster) como en su ubicación en el calendario. Efectivamente MLD se imparte de forma condensada en la segunda mitad del primer semestre del curso. Esto provoca una disminución en determinadas actividades temporales (por ejemplo, hay menos semanas donde está abierta la gamificación con Twitter) pero, por otra parte, al "competir" con menos asignaturas (el resto de asignaturas del plan de estudios del máster registra las mismas características, repartiéndose el semestre en dos mitades, de forma que mientras en grado DyP comparte calendario con un número equivalente al $50 \%$ de las asignaturas, MLD lo hace con tan solo un $25 \%$ de las mismas). Este factor podría afectar negativamente a las cifras obtenidas en los resultados de MLD, por lo que volveremos al mismo cuando evaluemos los resultados.

- Distintos pesos en las evaluaciones: aunque podríamos establecer criterios de comparación reduciendo diferencias considerando elementos que tienen pesos parecidos en una y otra asignatura (p.e. trabajo de asignatura en MLD vs peso de las prácticas en DyP), dado que nos centramos en la gamificación, cifraremos nuestro interés en estos valores (participación en foros, Twitter y debates) y la frecuencia con la que pueden ser recogidos (número de foros y debates, número semanas en las que la gamificación está activa en Twitter), sin considerar elementos tales como cuales de los casos que van asociados al debate tienen una nota específica, pues esto no afecta al modo en que se trata la gamificación en ellos. Vemos que, de nuevo, el ajustado calendario de la asignatura de Máster, provoca que aparezcan menos oportunidades de gamificación. Sin embargo, comprobaremos que estas oportunidades han sido más aprovechadas. Valga como muestra el que siendo muchos más los foros abiertos en poliformat para ofrecer espacio a la gamificación en la asignatura de grado, el número de foros realmente empleados por los alumnos es más reducido que el número de foros que emplean los alumnos de máster.

\subsection{Comparación:}

Los elementos que nos permitirán establecer comparaciones vienen de dos vías: la primera, la observación del profesoren el desarrollo de las distintas actividades de gamificación. La segunda, las estadísticas que la herramienta poliformat nos facilita sobre el acceso a las distintas herramientas que presenta.

(cc) EY-NC-ND 2018, UniversitatPolitècnica de València

Congreso IN-RED (2018) 


\section{Desarrollo de la innovación}

La experiencia, desarrollada en el ámbito temporal y docente que queda delimitado en el anterior apartado, no pudo ser aplicada de forma idéntica en número de actos a evaluar por la diferencia tan abultada en el lapso de tiempo y sesiones. Eso provocó que el número de elementos en la asignatura de máster, MLD, fuera significativamente más reducida (véase tabla 2). De todas formas, aunque los datos en bruto nos mostrarán que esta reducción en el número de eventos gamificables no parece provocar una abultada diferencia en favor de la participación de los alumnos de grado, normalizaremos estos datos buscando que las proporciones sean equilibradas, aportando un dato calculado que de la participación media por evento.

Con respecto al perfil de los alumnos participantes, cabe añadir a los datos objetivos recogidos en las tablas que los alumnos de DyP, cursada dentro del doble grado de Administración de Empresas más Informática, tiene un perfil que se diferencia del resto de alumnos que cursan DyP, en el segundo semestre, matriculados en el grado de informática. La diferencia más cuantificable la tenemos en su nota de acceso a la universidad (3,3 puntos mayor para los primeros), además de que cursan la materia en el primer semestre de su tercer curso, en lugar de en el segundo semestre de segundo curso. Sus notas son ostensiblemente más altas que la media (se triplica el porcentaje de sobresalientes, se duplica el de notables y se reduce a cero el número de suspensos y no presentados). Así como en otros grupos existe un número no numeroso pero ni mucho menos despreciable de alumnos que no realizan alguna actividad (un caso de aula, una práctica), esta circunstancia no se da en el grupo de doble grado, donde todos los participantes mostraron una constancia y un seguimiento de las clases absolutas.

Los datos que se muestran provienen de la observación directa del profesor, de su cuaderno de campo y preguntas realizadas a los alumnos y de la recogida automática de datos al final de la experiencia, gracias a las herramientas automatizadas de la plataforma docente.

La recogida manual de datos se efectuaba nada más cerrada una actividad (al final de un debate en aula, cuando concluía una semana en twitter -se consideraban activos los lapsos de sábado a viernes- y cuando se finalizaba un foro de debate (estos a priori permanecían abiertos durante una semana, pero cuando había interesados en participar, el plazo se alargaba al doble).

\section{Resultados}

Vamos a mostrar desglosados los datos obtenidos en cada una de las tres subactividades de gamificación y, a continuación, el uso que unos y otros alumnos hacen de las distintas herramientas y posibilidades de la plataforma, haciendo especial hincapié en las relacionadas con la gamificación estudiada y en los contenidos de la asignatura (todo el material de teoría se encontraba disponible en "contenidos").

Tabla 3. Uso de los foros para la gamificación, por parte de los alumnos.

\begin{tabular}{llc}
\hline Foros & DyP & MLD \\
\hline
\end{tabular}




\begin{tabular}{lcccc}
\hline Abiertos & 7 & \multicolumn{2}{c}{2} \\
Empleados & 1 & & 2 & $30 \%$ \\
Participación & 2 & $7 \%$ & 6 & $30 \%$ \\
Participación media por foro & 2 & $7 \%$ & 6 & Fuente: Elaboración propia \\
\hline
\end{tabular}

La lectura de esta primera tabla, referida a los foros, nos hace ver que, mientras en DyP se abren muchos más foros (por disponer de más semanas activas de clase), solo uno de ellos (de los siete abiertos) es realmente usado por los alumnos, en concreto por dos de ellos. En MLD, con un número muy inferior de foros (2) el uso es muy superior, hablemos de números brutos o de porcentajes.

Tabla 4. Debates realizados en clase y su cuantificación en la gamificación.

\begin{tabular}{llccc}
\hline Debates & DyP & \multicolumn{3}{c}{ MLD } \\
\hline Realizados & 7 & & 5 & $55 \%$ \\
Participación & 13 & $45 \%$ & 11 & $45 \%$ \\
Participación media por debate & 8 & $28 \%$ & 9 & Fuente: Elaboración propia
\end{tabular}

Observamos que tanto en la participación total (alumnos que han participado en algún debate) como en la que promedia el número de participantes por debate, el alumno de máster resulta más activo que el de grado.

Tabla 5. Actividad de los alumnos en Twitter, dentro de las actividades de gamificación.

\begin{tabular}{llllc}
\hline Twitter & DyP & \multicolumn{3}{c}{ MLD } \\
\hline Semanas de actividad & 10 & & 5 & $50 \%$ \\
Participación & 9 & $31 \%$ & 10 & $40 \%$ \\
Participación media por semana & 6 & $21 \%$ & 8 & Fuente: Elaboración propia \\
\hline
\end{tabular}

De igual manera que en los casos anteriores, podemos observa que el alumno de máster se muestra más activo.

Cerremos este apartado con una tabla que nos muestra la información sobre la gamificación en general, tanto con respecto a su actividad como sobre las notas máximas y mínimas alcanzadas. 
Tabla 6. Participación de los alumnos en actividades de gamificación: actividad y notas alcanzadas.

\begin{tabular}{llllll}
\hline Participación total & \multicolumn{2}{c}{ DyP } & \multicolumn{3}{c}{ MLD } \\
\hline $\begin{array}{l}\text { Alumnos participantes en al menos una } \\
\text { actividad }\end{array}$ & 22 & $76 \%$ & 12 & $60 \%$ \\
\hline $\begin{array}{l}\text { Alumnos que participan de las tres vías } \\
\text { Alumnos que participan en todas las ocasiones }\end{array}$ & 4 & 10 & $34 \%$ & 8 & $40 \%$ \\
Nota máxima obtenida por gamificación & 1,3 & & 0,9 & $30 \%$ \\
\hline \begin{tabular}{l} 
Nota mínima obtenida por gamificación \\
\hline
\end{tabular} & 0 & & 0 & \\
\hline
\end{tabular}

En la tabla anterior aparece una aparente contradicción que trataremos de aclarar. Si bien hemos visto hasta ahora que los alumnos de máster se mostraban más activos, el hecho de que el número de semanas de actividad de la asignatura de grado duplicara a la de máster provocó que un buen número de alumnos, quizá por probar, realizaran alguna actividad, si bien en muchos casos sin persistir en la experiencia. Vemos que los alumnos que no solo participan de todas las posibilidades, sino que "no se pierden una" (participan en todos los debates, foros, tuits), es más elevado en los alumnos de máster.

Otra posible sorpresa es la nota más alta alcanzada, más alta en DyP que en MLD. Esto tiene una doble explicación: de nuevo, el tiempo, que provoca que los interesados tengan más oportunidades para sacar una mejor nota, y por otra que, en este colectivo de alumnos excelentes, hubo dos de ellos que se tomaron muy en serio la competición que propuse: el primero en alcanzar la nota umbral (un punto) sería premiado con "una medalla de oro": 0,3 puntos extras. Ese dulce ofrecido al principio provocó hasta el final una verdadera carrera, con adelantamientos semana si, semana también.

Vamos ahora a ver los datos que la plataforma de forma automática nos recoge.

Tabla 7. Información ofrecida por la plataforma docente.

\begin{tabular}{lllll}
\hline Evento & DYP & MLD & $\begin{array}{l}\text { DYP por } \\
\text { alumno }\end{array}$ & $\begin{array}{l}\text { MLD } \\
\text { por } \\
\text { alumno }\end{array}$ \\
\hline Leer mensaje de foro & 15 & 50 & 0,52 & 2,50 \\
\hline Responder foro de mensaje & 2 & 12 & 0,07 & 0,60 \\
\hline Nuevo tema de foro & 1 & 10 & 0,03 & 0,50 \\
\hline Editar tema de un foro & 3 & 2 & 0,10 & 0,10 \\
\hline Acceso a una sección de contenidos & 2372 & 1520 & 81,79 & 76,00 \\
\hline
\end{tabular}




\begin{tabular}{|c|c|c|c|c|}
\hline Evento & DYP & MLD & $\begin{array}{l}\text { DYP por } \\
\text { alumno }\end{array}$ & $\begin{array}{l}\text { MLD } \\
\text { por } \\
\text { alumno }\end{array}$ \\
\hline Nuevo mensaje & 40 & 51 & 1,38 & 2,55 \\
\hline Responder mensaje & 11 & 7 & 0,38 & 0,35 \\
\hline Leer correo interno & 93 & 134 & 3,21 & 6,70 \\
\hline Leer recurso & 3174 & 2276 & 109,45 & 113,80 \\
\hline Acceso a calendario & & 42 & 0,00 & 2,10 \\
\hline Acceso a calificaciones & 51 & 31 & 1,76 & 1,55 \\
\hline Acceso a anuncios & 26 & 17 & 0,90 & 0,85 \\
\hline
\end{tabular}

En la información se añade una simple división, para saber cuántos de esos eventos se han producido por alumno. Observamos que en la única actividad de gamificación que puede seguirse desde la plataforma, la que está basada en el uso de foros, los números siguen indicando una mayor participación del alumno de MLD.

Otros datos se muestran como simple escenario. Por ejemplo, los relativos a los mensajes, consulta de calificaciones o lectura de los anuncios en el tablón. Como dato curioso, en DyP ningún alumno consultó el calendario de la plataforma.

De esta tabla podemos ver que el uso de los recursos y contenidos tienen ligeras variaciones en uno u otro sentido. Hay que indicar que este elemento no sirve más que como orientación muy superficial, pues el numero de recursos (documentos) y contenidos es inferior en máster frente a grado por el menor número de temas de una asignatura frente a la otra (menos temas, más complejos, pero con menos elementos a descarga o consulta, a pesar de que cada uno de ellos pueda ser de más tamaño). Por eso, excusamos el indicar mediante tablas el acceso a los contenidos y recursos clasificado por meses.

Por último, veremos a modo de comparativa las notas finales

Tabla 8. Notas finales.

\begin{tabular}{|c|c|c|c|c|}
\hline & DyP & DyP (\%) & MLD & $\begin{array}{l}\text { MLD } \\
(\%)\end{array}$ \\
\hline MH & 1 & $3,44 \%$ & 1 & $5 \%$ \\
\hline SB & 9 & $31,03 \%$ & 4 & $20 \%$ \\
\hline NT & 17 & $58,62 \%$ & 11 & $55 \%$ \\
\hline AP & 2 & $6,89 \%$ & 4 & $20 \%$ \\
\hline SP & 0 & $0 \%$ & 0 & $0 \%$ \\
\hline
\end{tabular}




\begin{tabular}{lllll}
\hline No presentados & 0 & $0 \%$ & 0 & $0 \%$ \\
PRES & 29 & & 20 & \\
NO PRES & 0 & & 1 & \\
NOTA MEDIA & 8,27 & $(10-5)$ & 7,85 & $(10-5,4)$ \\
\hline
\end{tabular}

Las notas, más altas en grado que en máster pueden deslucir lo anteriormente expuesto. Pero solo en apariencia. Porque si consideramos el alto número de alumnos excelentes que conformaban la matrícula, y que con las actividades programadas, no de forma extra, como la gamificación, sino las que estrictamente se enumeran en la guía docente, estamos ante un resultado que el docente esperaba desde los primeros días de clase. Por dar un ejemplo concreto, en la prueba concreta del primer caso que se evalúa en aula, que es además la primera actividad de la cual los alumnos obtienen una nota, y que en el desarrollo habitual de la asignatura (alumnos de grado) el número de suspensos ronda un 40\%. Con estos alumnos de doble grado, nadie suspendió, siendo las notas máximas las que alcanzaron ese $40 \%$.

\section{Conclusiones}

Hemos realizado una comparación con dos grupos de alumnos, unos cursando Máster y otros un doble grado, para intentar comprobar si el foco de su atención se centraba más o menos en una actividad que, siendo voluntaria, les permite subir nota en la asignatura.

Todo participante sabía que podía subir y bajar de la experiencia a voluntad. El docente siempre iba a considerar la nota que hubiera obtenido, fuera cual fuera el rango de fechas y actividades con el que las obtuviera.

Siendo actividades donde muchas veces el participante no obtiene nada (por definición solo se premiaba las mejores participaciones, y a veces quedaba desierto) la voluntad del alumno influye mucho más en su seguimiento que en las actividades ordinarias, de carácter obligatorio, con que la asignatura cuenta.

De los datos obtenidos observamos que, pese a la gran calidad del alumno de doble grado que era evaluado, los alumnos de máster, cuya diferencia con los anteriores en sentido positivo cabe cifrar en una mayor experiencia vital y laboral por su mayor edad, son más participativos. ¿Significa esto que esa "verdad no contrastada", que compartimos muchos docentes en charlas de pasillo, asumiendo una mayor maduración en el alumno de máster frente al alumno de grado es una realidad?. Dada la ausencia de datos a gran escala que permitan hacer una comparación y, obviamente, así mismo de la ausencia de perfiles psicológicos a los que acudir, no se puede dar una respuesta a esta pregunta. Pero lo que si se constata es una mayor participación en actividades de carácter voluntario, y un mayor uso de los recursos que desde la plataforma se ofertaba, por parte de los alumnos de máster. Para ser honrado, debo indicar que a título personal, sin ser los datos concluyentes, se subraya esa percepción íntima. 
Cabe añadir como una conclusión accesoria, que de estos resultados el docente obtiene una determinación: eliminar el uso de los foros para la gamificación, quedando descartados para posteriores ediciones por su muy bajo uso.

Para posteriores ediciones, queda el propósito de extender esta iniciativa a otras asignaturas de otras titulaciones y poder realizar una comparativa más amplia, al tiempo que aparece la idea de localizar elementos que puedan medir científicamente ese grado de maduración, y que puedan ser obtenidos de forma no intrusiva del alumnado..

\section{Referencias}

Boza Carreño, A.; Tocano Cruz, M. De La O. (2012) "Motivos, actitudes y estrategias de aprendizaje: aprendizaje motivado por alumnos universitarios". Profesorado, 16(1): 125142 (2012). [http://hdl.handle.net/10481/22994] [Consulta: 10 de marzo 2018]

Creed, P.A.; Hughes, T. (2012)."Career development strategies as moderators between career compromise and career outcomes in emerging adults". En Journal of Career Development, 40(2), 146-163. doi: 10.1177/0894845312437207[Consulta: 10 de marzo 2018]

Deterding, S., Dixon, D., Khaled, R., \& Nacke, L. (2011). "From game design elements to gamefulness: Defining "gamification."' In Proceedings of the 15th International Academic MindTrek Conference: Envisioning Future Media Environments (pp. 9-15). New York: ACM.

Donoso, S. (1998)."La reforma educacional y el sistema de selección de alumnos a las universidades: impactos y cambios demandados" Estudiospedagógicos.n.24 pp. 7-30 Valdivia. http://dx.doi.org/10.4067/S0718-07051998000100001. [Consulta: 10 de marzo 2018]

Fuchs, M., Fizek, S., Ruffino P., Schrape, N. (2014) Rethinking Gamification. Lüneburg, Alemania: Meson press.

Kinash, S.; Crane, L.; Capper, J., Young, M.; Stark, A. (2017). "When do university students and graduates know what careers they want: A research-derived framework." Journal of Teaching and Learning for Graduate Employability, 8(1), 3-21.21 https://doi.org/10.21153/jtlge2017vol8no1art584 [Consulta: 10 de marzo 2018]

Oltra Gutiérrez, J.V.; Garrigós-Simón, F.J.; Narangajavana, Y. (2016). "Introducción de actividades de Gamificación en una asignatura humanista para informáticos”. En IN-RED 2016. Valencia: Universitat Politècnica de Valencia. DOI: http://dx.doi.org/10.4995/INRED2016.2016.4430. [Consulta: 10 de marzo 2018]

Oltra Gutiérrez, J.V.; Garrigós-Simón, F.J.; Narangajavana, Y.; Montesa Andrés, J.O. (2016). "Gamificación dentro y fuera del aula: una experiencia. Deontología y Profesionalismo para informáticos.”. En INNODOCT 2016. Valencia: Universitat Politècnica de Valencia PP. 559-566

Pelling, N. (2011). "The (short) prehistory of gamification. Funding Startups (\& other impossibilities)". Haettu ,7, 2013.

Werbach, K. (2014). "(Re) Defining Gamification: A Process Approach. In Persuasive Technology" (pp. 266-272). Springer International Publishing.

(cc) EY-NC-ND 2018, UniversitatPolitècnica de València

Congreso IN-RED (2018) 